\title{
Velocidade de marcha, quedas e sua autoeficácia como modelos preditores de fragilidade
}

\author{
Lilian Atalaia-Silva, ${ }_{1}^{1}$ Kelly C. Atalaia-Silva, ${ }^{2}$ Eliane F.C. Banhato, ${ }^{3}$ Danielle V. Guedes, ${ }^{4}$ Roberto A. \\ Lourenço, ${ }^{5}$ Cláudia Helena C. Mármora ${ }^{6}$
}

\begin{abstract}
Resumo
Introdução: A fragilidade é uma síndrome que acomete idosos mais vulneráveis, predispondo à incapacidade funcional e até ao óbito. O objetivo do presente trabalho foi investigar se o modelo composto por velocidade de marcha, número de quedas e sua autoeficácia poderia explicar o desfecho fragilidade em idosos. Materiais e Métodos: Foi realizado um estudo transversal em uma amostra de idosos da comunidade da cidade de Juiz de Fora (MG). Foram utilizados os instrumentos Falls Efficacy Scale International (FES-I); cálculo da velocidade de marcha e questionário sobre quedas. Resultados: A amostra foi composta por 427 idosos, sendo 40 considerados frágeis pelo fenótipo de fragilidade. O beta encontrado foi de 2,259 e todas as variáveis foram estatisticamente significativas ( $\mathrm{p}<0,001)$. OR de Nagelkerke foi de 0,468 , indicando que o modelo, composto por velocidade de marcha, FES-I e número de quedas, explica 47\% da variável dependente: fragilidade. Discussão: As variáveis analisadas mostraram-se boas preditoras da síndrome da fragilidade. Com base nesses dados, poderão ser desenvolvidos modelos de diagnóstico e cuidado para o idoso frágil (detecção precoce e tratamento) e para o idoso não frágil (prevenção primária) com a utilização de instrumentos validados para o país, considerando que ainda não existem trabalhos dessa natureza e com essa abrangência na realidade brasileira. Conclusão: Velocidade de marcha, número de quedas e FES-I parecem ser preditores importantes do desfecho fragilidade em uma amostra representativa de idosos comunitários.
\end{abstract}

Descritores: Fragilidade; Marcha; Cognição; Acidentes por quedas.

\section{Abstract \\ Gait speed, falls and fall self-efficacy as predictors models of frailty}

Introduction: Frailty is a syndrome that affects vulnerable elderly, predisposing to functional disability and even death. The aim of the present study was to determine if gait speed, number of falls and self-efficacy could predict fragility in the elderly. Materials and Methods: A cross-sectional study was carried out on a sample of elderly people from the city of Juiz de Fora (MG). The instruments used were: Falls Efficacy Scale International (FES-I); gait speed and the questionnaire about falls. Results: The sample was comprised of 427 elderly people, 40 of whom were vowed by the fragility phenotype. Beta was 2.259 and all variables were statistically significant ( $p$ $<0.001$ ). Nagelkerke's R was 0.468 , indicating that the model,
1. Departamento de Fisioterapia. Centro Universitário Estácio Juiz de Fora. Juiz de Fora, MG, Brasil.

2. Centro de Ciências da Saúde. Universidade Federal do Recôncavo da Bahia. Santo Antonio de Jesus, BA, Brasil.

3. Departamento de Psicologia. Centro de Ensino Superior de Juiz de Fora. Juiz de Fora, MG, Brasil.

4. Departamento de Psicologia. Universidade Federal de Juiz de Fora. Juiz de Fora, MG, Brasil.

5. Departamento de Medicina Interna. Faculdade de Ciências Médicas da Universidade do Estado do Rio de Janeiro. Rio de Janeiro, RJ, Brasil.

6. Faculdade de Fisioterapia. Universidade Federal de Juiz de Fora. Juiz de Fora, MG, Brasil.

*Endereço para correspondência:

Centro Universitário Estácio Juiz de Fora. Faculdade de Fisioterapia.

Avenida Presidente João Goulart, 600

Juiz de Fora, MG, Brasil. CEP: 36030-900.

E-mail: lilian.atalaia.fisio@gmail.com

Revista HUPE, Rio de Janeiro, 2018;17(2):65-70

doi: $10.12957 /$ rhupe.2018.40862

Recebido em 07/05/2018. Aprovado em 21/10/2018.

composed of gait speed, FES-I and number of falls, explains $47 \%$ of the dependent variable: fragility. Discussion: The analyzed variables seem to be good predictors of the fragility syndrome. Based on these results, diagnostic and care models for the fragile elderly (early detection and treatment) and for the non-fragile elderly (primary prevention) can be developed with the use of validated instruments for Brazil, considering that there are still few researches with this scope in Brazil. Conclusion: Gait speed, number of falls and FES-I seem to be important predictors of frailty in a representative sample of community-dwelling elderly.

Keywords: Frailty; Gait; Cognition; Accidental falls.

\section{Resumen}

\section{Velocidad de marcha, caídas y su autoeficacia como} modelos predictores de fragilidad

Introducción: La fragilidad es un síndrome que ocurre em ancianos vulnerables, conllevando desde una discapacidad funcional hasta la muerte. El objectivo del presente trabajo 
ha sido evaluar si el modelo conformado por velocidad de la marcha, número de caídas y su autoeficacia podría predecir el síndrome de fragilidad en los ancianos. Materiales y métodos: Fue realizado un estudio transversal em una muestra de ancianos de la comunidad de Juiz de Fora (MG). Han sido utilizados los instrumentos Falls Efficacy Scale International(FES-I); cálculo de velocidad de la marcha e inventario de evaluación de caídas. Resultados: La muestra fue conformada por 427 ancianos, y dentre ellos, 40 han sido considerados frágiles por el fenotipo de fragilidad. La beta fue de 2,259 y todas las variables tuvieron significación estadística ( $\mathrm{p}<0,001)$. La R de Nagelkerke fue de 0,468 , lo que apunta para el hecho de que el modelo, conformado por velocidad de la marcha, número de caídas y FES-I explica el 47\% de la variable dependiente fragilidad. Discusión: Las variables parecen ser buenas predictoras del síndrome de fragilidad. A partir de estos datos, futuros investigadores podrán desarrollar modelos diagnósticos y de cuidado para el anciano frágil (detección precoz y tratamiento) y para el anciano no frágil (prevención primaria) con la utilización de instrumentos validados para el país, puesto que todavia no existen trabajos de esta naturaleza y con esta amplitud en la realidad brasilenã. Conclusión: Velocidad de la marcha, número de caídas y FES-I suelen predecir el síndrome de fragilidad en una población de ancianos que viven en la comunidad.

Palabras clave: Fragilidad; Marcha; Cognición; Accidentes por caídas.

\section{Introdução}

Aproximadamente 30\% das pessoas com 65 anos ou mais caem a cada ano, e esta taxa tende a aumentar com o passar dos anos. ${ }^{1}$ Cerca de $13 \%$ são "caidores" (fallers) recorrentes, o que favorece a institucionalização de idosos que previamente eram independentes. ${ }^{2} \mathrm{~A}$ queda em idosos pode gerar sérios prejuízos à qualidade de vida, tais como imobilidade, dependência dos familiares, diminuição da funcionalidade, depressão, internação e complicações advindas de cirurgia. ${ }^{3}$ Nos casos mais graves, a queda pode levar à morte. Porém, as consequências físicas nem sempre são os fatores mais devastadores para o idoso.

O trauma psicológico é bastante comum em episódios de quedas e é geralmente expresso pelo medo de cair, independente de trauma físico e/ ou limitação funcional. ${ }^{4,5}$ Esse medo pode levar a comportamentos cautelosos devido à perda da confiança (especialmente do senso de autoeficácia) no equilíbrio e na mobilidade corporal, favorecendo o declínio funcional e consequentemente elevando o risco de quedas futuras. ${ }^{6}$

A marcha é um pré-requisito para a manutenção da independência nas atividades básicas de vida diária, e distúrbios de marcha podem tornar o idoso mais

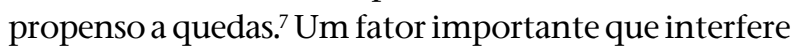
no desempenho da marcha é a velocidade, considerada o parâmetro isolado que melhor a representa. ${ }^{8,9} \mathrm{~A}$ velocidade tende a diminuir com o passar dos anos, cerca de 1 a $2 \%$ por década até os 60 anos de idade, e depois aproximadamente $7 \%$ por década. ${ }^{10}$ Diversos estudos têm mostrado relação entre baixa velocidade de marcha e perda de funcionalidade.,211,12
As quedas podem ter como causa diversos fatores, dentre eles destacam-se a velocidade de marcha e a perda de força muscular. ${ }^{13}$ Tais itens fazem parte de um critério para identificar uma síndrome recentemente caracterizada entre os idosos, chamada Síndrome da Fragilidade. A fragilidade é considerada altamente prevalente no processo de envelhecimento, incluindo declínio associado à idade, perda de massa corporal, resistência, equilíbrio e desempenho na marcha e baixa atividade, compondo o ciclo da fragilidade. ${ }^{14-16}$

No Brasil há uma carência de estudos que investiguem a relação entre quedas, velocidade de marcha e autoeficácia para quedas em idosos, especialmente na população considerada frágil. Diante disso, surge a necessidade de se estudar tais características a fim de não só retratar o idoso frágil, mas também e principalmente de rastrear idosos que podem se tornar frágeis, promovendo medidas de intervenção e acompanhamento desses indivíduos.

Nesta direção, o objetivo do trabalho foi investigar se o modelo composto por velocidade de marcha, número de quedas e sua autoeficácia poderia explicar o desfecho fragilidade em idosos.

\section{Materiais e métodos}

A pesquisa fez parte do projeto rede FIBRA Brasil, com apoio do Conselho Nacional de Desenvolvimento Científico e Tecnológico (CNPq) e respeitando todos os princípios éticos. ${ }^{12}$ Participaram do estudo 427 idosos comunitários com idade igual ou superior a 65 anos. 


\section{Instrumentos}

Os seguintes instrumentos foram utilizados:

- A Escala de autoeficácia para quedas, Falls Efficacy Scale International (FES-I), avalia a preocupação com a possibilidade de cair ao realizar 16 atividades de vida diária, com respectivos escores de um a quatro. $^{13} \mathrm{O}$ escore total varia de 16 (ausência de preocupação) a 64 (preocupação extrema).

- Velocidade de marcha: para o cálculo da velocidade foi utilizado uma fita para delimitar no solo um espaço de 4,6 metros. O tempo que o idoso percorreu foi mensurado por um cronômetro. Esse procedimento foi realizado por três vezes, calculando-se a velocidade média da marcha.

- Quedas: foi empregado o questionário sobre histórico de quedas contendo duas perguntas: "No último ano o(a) senhor(a) sofreu alguma queda? Se sim, quantas?"

A partir dos dados supracitados, a amostra foi dividida em idosos frágeis e não frágeis conforme o fenótipo de fragilidade desenvolvido por Fried e colaboradores, composto pelas seguintes características: perda de peso não intencional, velocidade de marcha, força de preensão palmar, baixo gasto quilocalórico e fadiga. ${ }^{15}$

Cabe ressaltar que para o indivíduo ser considerado frágil era necessário possuir pelo menos três critérios propostos por Fried. Neste sentido, os idosos que possuíssem 1 ou 2 critérios não eram frágeis, mas considerados pré-frágeis. Tal denominação indica que a chance de se tornarem frágeis era maior em relação aos que não se enquadravam em nenhum critério.

\section{Análise estatística}

Primeiramente foi realizado o teste t de Student para amostras independentes com o intuito de comparar as diferenças de média da velocidade da marcha, FES-I e número de quedas entre homens e mulheres e também entre frágeis e não frágeis. Posteriormente, realizou-se a correlação de Pearson entre as variáveis velocidade de marcha, autoeficácia para quedas e evento quedas na amostra total e ao se estratificar pelo fenótipo de fragilidade. A última etapa compreendeu a realização de uma regressão logística, método enter, para calcular a variável dependente ( $\mathrm{VD}=$ sujeitos frágeis $\mathrm{x}$ não frágeis), investigando se o modelo composto por velocidade de marcha, quedas e autoeficácia poderia ser preditor do desfecho fragilidade.

\section{Resultados}

A amostra foi composta por 427 idosos, sendo $70 \%$ mulheres, a idade variou de 65 a 97 anos $(74,6 \pm 7)$, $72 \%$ se autodeclararam brancos, $10 \%$ negros e $18 \%$ mulatos, caboclos ou pardos. Quanto ao estado civil, $48 \%$ são casados ou vivem com companheiro(a), $10 \%$ são solteiros, $7 \%$ são divorciados ou separados e $36 \%$ são viúvos. A média de escolaridade foi de 5,8 anos ( $\mathrm{DP}=7,6$ ). A frequência de quedas entre os idosos foi de $33 \%$. Ao estratificar a amostra segundo sexo, verificou-se que as mulheres apresentaram maior número de quedas, tiveram mais medo de cair e desempenho inferior na velocidade de marcha quando comparadas com os homens (Tabela 1).

A fim de correlacionar o número de quedas com velocidade de marcha e sua autoeficácia na amostra total, constatou-se correlação estatisticamente significativa entre todas estas variáveis (Tabela 2).

Ao se estratificar a amostra pelo fenótipo de fragilidade, 40 sujeitos foram considerados frágeis (média de idade de 79,05 $\pm 7,03$ ). Houve diferença estatisticamente significativa em relação ao número de quedas, à autoeficácia (FES-I) e à velocidade média de marcha (Tabela 3).

Entre os indivíduos não frágeis, houve correlação estatisticamente significativa entre número de quedas e FES-I e entre FES-I e velocidade de marcha, mas não houve correlação estatisticamente significativa entre número de quedas e velocidade de marcha (Tabela 4).

Foi encontrado na regressão logística beta igual a 2,259, e todas as variáveis foram estatisticamente significativas $(p<0,001)$. O R de Nagelkerke foi de 0,468 , indicando que o modelo composto por velocidade de marcha, FES-I e número de quedas, explica $47 \%$ da variável dependente: fragilidade.

\section{Discussão}

No presente estudo, o número de indivíduos caidores foi semelhante ao encontrado por Perracini \& Ramos e Liu-Ambrose., ${ }^{1,2}$ Em geral, é conhecido na literatura que as mulheres caem mais que os homens; $74 \%$ dos idosos que sofreram pelo menos uma queda nos últimos 12 meses são mulheres, dado que condiz com os trabalhos de Boyd \& Stevens. ${ }^{17}$ Já a autoeficácia de quedas, mensurada pela FES-I, mostrou uma média significativamente superior nas mulheres $(\mathrm{p}<0,001)$, assim como no estudo de Yardley. ${ }^{18}$ Talvez o fato de sofrer mais quedas faça com que as idosas tenham maior preocupação com a possibilidade de cair novamente, 


\section{Artigo original}

Tabela 1. Quedas, FES-I e velocidade de marcha por sexo

\begin{tabular}{|c|c|c|c|c|c|c|}
\hline & Sexo & $\mathrm{N}$ & Média & Desvio Padrão & Teste $\mathrm{t}$ & $\mathrm{p}$ \\
\hline \multirow{2}{*}{ Número de Quedas } & Masculino & 130 & 0,41 & 0,78 & \multirow{2}{*}{$-2,1$} & \multirow{2}{*}{$0,037^{*}$} \\
\hline & Feminino & 297 & 0,66 & 1,29 & & \\
\hline \multirow{2}{*}{ FES-I } & Masculino & 128 & 24,14 & 7,79 & \multirow{2}{*}{$-4,5$} & \multirow{2}{*}{$0,001^{*}$} \\
\hline & Feminino & 296 & 28,04 & 8,29 & & \\
\hline \multirow{2}{*}{$\begin{array}{l}\text { Média da Velocidade } \\
\text { de Marcha }\end{array}$} & Masculino & 128 & 1,96 & 0,58 & \multirow{2}{*}{3,1} & \multirow{2}{*}{$0,002^{*}$} \\
\hline & Feminino & 296 & 1,75 & 0,63 & & \\
\hline
\end{tabular}

Tabela 2. Correlações da amostra total

\begin{tabular}{lcccc}
\hline & & Número de Quedas & Média da Velocidade de Marcha & FES-I \\
\hline Número de Quedas & $r$ & 1 & $-0,11^{*}$ & $0,14^{*}$ \\
& $p$ & & 0,019 & 0,003 \\
Média da Velocidade de & $r$ & $-0,11^{*}$ & 1 & $-0,33^{*}$ \\
Marcha & $p$ & 0,01 & $-0,33^{*}$ & 0,001 \\
FES-I & $r$ & $0,14^{*}$ & 0,001 & 1 \\
& $p$ & 0,003 & &
\end{tabular}

Tabela 3. Quedas, FES-I e velocidade de marcha entre frágeis e não frágeis

\begin{tabular}{|c|c|c|c|c|c|}
\hline & Grupo & Média & Desvio Padrão & Teste $\mathrm{t}$ & $\mathrm{p}$ \\
\hline \multirow{2}{*}{ Número de Quedas } & Frágeis & 1,23 & 1,45 & \multirow{2}{*}{3,66} & \multirow{2}{*}{$0,001^{*}$} \\
\hline & Não frágeis & 0,52 & 1,12 & & \\
\hline \multirow{2}{*}{ FES-I } & Frágeis & 35,12 & 9,68 & \multirow{2}{*}{6,95} & \multirow{2}{*}{$0,001^{*}$} \\
\hline & Não frágeis & 26,00 & 7,69 & & \\
\hline \multirow{2}{*}{$\begin{array}{l}\text { Média da Velocidade } \\
\text { de Marcha }\end{array}$} & Frágeis & 1,02 & 0,47 & \multirow{2}{*}{$-9,28$} & \multirow{2}{*}{$0,001 *$} \\
\hline & Não frágeis & 1,90 & 0,58 & & \\
\hline
\end{tabular}

Tabela 4. Correlações entre indivíduos não frágeis

\begin{tabular}{lcccc}
\hline & & Número de Quedas & Média da Velocidade de Marcha & FES-I \\
\hline Número de Quedas & $r$ & 1 & $-0,49$ & $0,123^{*}$ \\
& $p$ & & 0,338 & 0,016 \\
Média da Velocidade de & $r$ & $-0,49$ & 1 & $-0,277^{*}$ \\
Marcha & $p$ & 0,338 & $-0,277^{*}$ & 0,001 \\
FES-I & $r$ & $0,123^{*}$ & 0,001 & 1 \\
& $p$ & 0,016 & & \\
\hline
\end{tabular}


porém cabe ressaltar que a autoeficácia para quedas pode ser superestimada pelos homens, uma vez que, culturalmente, estes não expressam tanto seus sentimentos e vulnerabilidades, principalmente em relação ao medo. ${ }^{13,19}$

No teste de velocidade de marcha, o sexo feminino apresentou um desempenho inferior, caracterizado por velocidade média igual a $1,75 \mathrm{~m} / \mathrm{s}$, enquanto os homens alcançaram média de $1,96 \mathrm{~m} / \mathrm{s}$. Tais dados estão em concordância com os achados de Melo. ${ }^{20}$ No trabalho de Abreu \& Caldas constatou-se que o grupo de menor velocidade de marcha apresentou uma incidência maior de quedas, situação também encontrada indiretamente no presente estudo, em que as mulheres possuem velocidade mais lenta e caem mais do que os homens. ${ }^{21}$

Na amostra total houve associação estatisticamente significativa entre velocidade de marcha, FES-I e número de quedas. Esses dados estão em concordância com estudo de Camargos que, apesar de a velocidade não ter sido seu objeto de pesquisa, encontrou associação estatisticamente significativa entre FES-I e quedas. ${ }^{12}$

A correlação entre FES-I, velocidade de marcha e quedas no grupo de frágeis não foi estatisticamente significativa. Tal fato talvez se justifique pelo pequeno número de indivíduos frágeis $(\mathrm{n}=40)$. No entanto, em sujeitos não frágeis, cujo número é expressivamente maior ( $n=384)$, houve correlação estatisticamente significativa entre número de quedas e FES-I e entre FES-I e velocidade de marcha, mas não entre número de quedas e velocidade de marcha. Em relação a estes aspectos é possível constatar que a preocupação com a possibilidade de cair interfere muito mais na velocidade de marcha do que propriamente no número de quedas. Scheffer realizou uma revisão sistemática a respeito da prevalência do medo de cair e constatou que 50\% das pessoas que possuíam medo de cair não haviam tido a experiência de uma queda anteriormente. ${ }^{22} \mathrm{O}$ medo de cair está fortemente associado ao equilíbrio e problemas na marcha, principalmente na alteração da velocidade. ${ }^{5,19,23-25}$

\section{Conclusão:}

Ao se investigar se o modelo composto por velocidade de marcha, número de quedas eFES-I poderia explicar o desfecho fragilidade, verificou-se que as variáveis supracitadas, em conjunto, explicam 47\% dos casos de fragilidade, demonstrando-se boas preditoras do desfecho mencionado. Com base nesses dados, poderão ser desenvolvidos modelos de diagnóstico e cuidado para o idoso frágil (detecção precoce e tratamento) e para o idoso não frágil (prevenção primária) com a utilização de instrumentos validados para o país, considerando que ainda não existem trabalhos dessa natureza e com essa abrangência na realidade brasileira.

\section{Referências}

1. Perracini MR, Ramos LR. Fatores associados a quedas em uma coorte de idosos residentes na comunidade. Rev Saúde Pública. 2002;36(6):709-16

2. Liu-Ambrose T, Davis JC, Nagamatsu LS, et al. Changes in executive functions and self-efficacy are independently associated with improved usual gait speed in older women. BioMed Central Geriatrics. 2010;10(25):1-8.

3. El-khoury F, Cassou B, Charles MA, et al. The effect of fall induced injuries in Community dwelling older adults: systematic review and meta-analysis of randomised controlled trials. BMJ. 2013;347:f6234. doi: 10.1136/bmj.f6234.

4. Tinetti ME, Mendes de Leon CF, Doucette JT, et al. Fear of falling and fall related efficacy in relationship to functioning among community-living elders. J Gerontol. 1994 May;49(3):M140-7.

5. Reelick MF, Van lersel MB, Kessels RP, et al. The influence of fear of falling on gait and balance in older people. Age and Ageing. 2009;38:435-440.

6. Lachman ME, Howland J, Tennstedt S, et al. Fear of falling and activity restriction: the survey of activities and fear of falling in the elderly (SAFE). J Gerontol B Psychol Sci Soc Sci. 1998;53(1):P4350.

7. Ferrucci L, Guralnik JM, Salive ME, et al. Effect of age and severity of disability on short-term variation in walking speed: the women's health and aging study. J Clin Epidemiol. 1996;49(10):1089-96.

8. Liu-Ambrose T, Nagamatsu LS, Graf P, et al. Resistance Training and executive functions: a 12-month randomised controlled trial. Arch Intern Med. 2010;170(2):170-8. doi: 10.1001/ archinternmed.2009.494.

9. Friedman PJ, Richmond DE, Baskett JJ. A prospective trial of serial gait speed as a measure of rehabilitation in the elderly. Age and Ageing 1998;17:227-35

10. Souza N, Mendes R, Silva A, et al. Combined exercise is more effective than aerobic exercise in the improvement of fall risk factors: a randomized controlled trial in community-dwelling older men. Clinical Rehabilitation. 2017;31(4):478-486.

11. Verghese J, Mahoney J, Ambrose AF, et al. Effect of cognitive remdiation on gait in sedentary seniors. Journal of Gerontology: Medical Sciences 2010;65A(12):1338-1343.

12. Lourenço RA, Moreira VLG, Banhato EFC, et al. Prevalence of frailty and associated factors in a community-dwelling older people cohort living in Juiz de Fora, Minas Gerais, Brazil: Fibra-JF Study. Ciênc Saúde Coletiva. 2019;24(1):35-44.

13. Camargos FF, Dias RC, Dias JM, et al. Cross-cultural adaptation and evaluation of the psycho $\neg$ metric properties of the Falls Efficacy Scale-Inter $\neg$ national Among Elderly Brazilians (FES-IBRAZIL). Rev Bras Fisioter. 2010;14:237-43.

14. Liu-Ambrose T, Nagamatsu LS, Graf P, et al. Resistance Training and executive functions: a 12-month randomised controlled trial. Arch Intern Med. 2010;170(2):170-8. doi: 10.1001/ archinternmed.2009.494.

15. Fried LP, Tangen CM, Walston J, et al. Frailty in Older Adults: 


\section{Artigo original}

Evidence for a Phenotype. J Gerontol A Biol Sci Med Sci. 2001 Mar;56(3):M146-56.

16. Rockwood K, Howlett SE, MacKnight C, et al. Prevalence, attributes, and outcomes of fitness and frailty in communitydwelling older adults: report from the Canadian Study of Health Study and Aging. J Gerontol A Biol Sci Med Sci. 2004 Dec;59(12):1310-7.

17. Boyd R, Stevens JA. Falls and fear of falling: burden, beliefs and behaviours. Age and Ageing. 2009;38:423-428.

18. Yardley L, Beyer N, Hauer K, et al. Development and initial validation of the Falls Efficacy Scale-International (FES-I). Age and ageing. 2005;34:614-9.

19. Vellas BJ, Wayne SJ, Romeo LJ, et al. Fear of falling and restriction of mobility in elderly fallers. Age and ageing. 1997;26:189-93.

20. Melo DM. Fragilidade, desempenho de atividades avançadas de vida diária saúde percebida em idosos atendidos em ambulatório de geriatria. Campinas. Dissertação [Mestrado em Gerontologia] - Faculdade de Ciências Medicas da Universidade Estadual de Campinas - UNICAMP; 2009.

21. Abreu SSE, Caldas, CP. Velocidade de marcha, equilíbrio e idade: um estudo correlacional entre idosas praticantes e idosas não praticantes de um programa de exercícios terapêuticos. Rev Bras Fisioter. 2008;12(4):324-30.

22. Scheffer AC, Schuurmans J, Dijk NV, et al. Fear of falling: measurement strategy, prevalence, risk factors and consequences among older persons. Age Ageing. 2008;37(1):1924. doi: 10.1093/ageing/afm169. Review.

23. Wurzer B, Waters DL, Hale LA, et al. Long-term participation in peer-led fall prevention classes predicts lower fall incidence. Arch Phys Med Rehabil. 2014 Jun;95(6):1060-6. doi: 10.1016/j. apmr.2014.01.018. Epub 2014 Feb 4.

24. Taylor D, Hale L, Schluter P, et al. Effectiveness of tai chi as a community-based falls prevention intervention: a randomized controlled trial. J Am Geriatr Soc. 2012 May;60(5):841-8. doi: 10.1111/j.1532-5415.2012.03928.x.

25. Granacher U, Muehlbauer T, Gollhofer A, et al. Evidencebased and evidence-inspired: an intergenerational approach in the promotion of balance and strength for fall prevention. Gerontology. 2011;57(5):424-6. doi: 10.1159/000322149. 